Pure Appl. Chem., Vol. 75, No. 5, pp. 609-619, 2003.

(C) 2003 IUPAC

\title{
Toward replicatable, multifunctional, nanoscaffolded machines. A chemical manifesto*
}

\author{
Guenter von Kiedrowski ${ }^{\ddagger}$, Lars-Henning Eckardt, Kai Naumann, \\ Wolf Matthias Pankau, Malte Reimold, and Michael Rein

\begin{abstract}
Chair of Organic Chemistry I, Bioorganic Chemistry, Ruhr-University Bochum NC2/171, Universitaetsstrasse 150, 44780 Bochum, Germany
\end{abstract}

\begin{abstract}
Self-replicating nanorobots were foreseen in technological dreams and visions whose scientific ground found solid criticism from chemical and physical reasoning. If one, however, views such constructs as three-dimensionally defined noncovalent nanoscaffolding of a multitude of modular functions whose array is replicatable in a nonautonomous way, many pieces of technology needed for their implementation became recently available. Gold cluster-labeled molecules were remotely controlled by $\mathrm{GHz}$ radio frequency causing local and selective inductive heating, and monoconjugable thermostable gold clusters will become commercially available soon. Charged molecules were electrophoretically steered and manipulated on the surface of microelectrode array chips. Surface-promoted replication and exponential amplification of DNA analogs (SPREAD) may find particular applications for the cloning and copying of informational nanostructures on the surface of such chips. Synthetic trisoligonucleotidyl junctions were reported as covalent building blocks for noncovalent DNA nanostructures, and it was shown that kinetic control during noncovalent synthesis favors small and defined nanostructures instead of polymeric networks. Very recently, it was demonstrated that functionalized DNA nanoscaffolds with stiff tensegrity such as tetrahedra self-assemble from maximally instructed sets of 3- or [3+1]-arm junctions, and that the connectivity information in such nanoscaffolds can be copied. The implications of these developments are discussed with respect to a possible implementation scheme for the issue of the title.
\end{abstract}

\section{INTRODUCTION}

The paradigm of material production in chemistry is synthesis; the paradigm of material production in biology is growth, based on self-replication and molecular information processing. Material properties in physics and chemistry arise by design and engineering; material properties in biology emerge in the process of natural evolution. The gap between synthesis and design on the chemical and physical side and growth and evolution on the biological side calls for a new generation of technologies that aim to combine the advantages of both the man-made and the natural strategy. This is a particular challenge, however, also a particular opportunity for technologies in the field of nanosciences. In the industrial context of material sciences, there is a strong argument against the mass production of highly complex and intelligent materials based on nonstandard polymers. The argument is simply based on the cost of

\footnotetext{
*Plenary lecture presented at the $16^{\text {th }}$ International Conference on Physical Organic Chemistry (ICPOC-16): Structure and Mechanism in Organic Chemistry, San Diego, California, USA, 4-9 August 2002. Other presentations are published in this issue, pp. 541-630.

${ }^{\ddagger}$ Corresponding author
} 
synthesis. It is, however, getting weaker, once production technologies become available that are either based on biological systems or mimic the latter systems in their elementary ability to replicate and to grow. Replicatable nanomachinery [1], known as nanorobots in the public, were seen as the Holy Grail of nanotechnology, and medical applications of the latter were envisioned to yield a new generation of drugs that carry the functionality of performing complex tasks in the human body [2]. However, the scientific concepts behind the schemes proposed for the implementation of nanorobots found solid criticism from chemical and physical reasoning [3,4]. Central to the criticism is the concept of a nanomechanical assembler that enables the manufacturing of nanomachines including itself in an atom-by-atom fashion. If nanomechanical fabrication of nanomachines is, however, replaced by their self-assembly from modular parts in the sense of a molecular self-instruction process, the creation of replicatable nanomachines seems to be feasible today.

\section{MANIFESTO FOR NANOROBOT IMPLEMENTATION}

We propose a scheme for the implementation of a prototype generation of replicatable nanomachines that is based on:

- noncovalent informational nanoscaffolding and arraying a multitude of modular functions expressed by organic, inorganic, or biological components in a defined 3D arrangement;

- the self-assembly of such objects from synthetic 3-arm junctions where each arm is a composite/conjugate from an informational molecule such as DNA, RNA, or a synthetic mimic of the latter and an individual functional module;

- $\quad$ tensegrity and maximal instruction as the keys for nanoarchitectural control;

- the copying of connectivity information in such junctions as the key for nanomachine replication,

- the controlled cloning and copying of junctions on the surface of electronically addressable chips by means of a chip variant of the procedure published as "surface-promoted replication and exponential amplification of DNA analogs" (SPREAD);

- the usage of linear conjugates between oligonucleotides and single modular functions as basic construction elements for the fabrication of functionalized 3-arm junctions and the latter's selfassembly into multifunctionalized nanoscaffolded machines;

- the directed evolution of replicatable nanomachines by a variant of the SELEX-procedure starting from random connectivity information;

- the usage of such nanomachines for the probing of nanoepitopes on the surfaces of biological cells;

- the external control of the operation of such nanomachines by $\mathrm{GHz}$ radio frequency magnetic field inductive heating of metal clusters attached to such constructs.

Several key steps of the proposed technology have been realized already in laboratories, including ours. However, the full potential of replicatable nanoscaffolded, multifunctional materials and machines can only be exploited if the pieces of technology finally fit together. This is why we think that a kind of open project in chemistry may be the most suitable form here.

\section{Noncovalent informational nanoscaffolding}

Noncovalent informational nanoscaffolding was demonstrated in a number of ways using linear DNA as informational templates [5]. Conjugates of oligonucleotides with nanoparticles, dyes, proteins, and other functions were hybridized with the oligonucleotide part to the complementary stretch of the linear template. The array of function modules was studied with various techniques including TEM, STM, and AFM. Defined 3D nanoarchitectures are, however, difficult to achieve following this strategy, as linear assemblies can fold in many different ways. 


\section{Self-assembly from synthetic 3-arm junctions}

Nadrian Seeman has demonstrated the feasibility of synthesizing 3D nanoarchitectures such as cubes [6] from DNA as the construction material. Seeman's nanoobjects are covalent structures, whose synthesis started from sets of three linear oligonucleotides that self-assembled into noncovalent 3-way junctions (Fig. 1). These junctions have sticky ends that were joined and then ligated to form covalent nanostructures. Recently, technologies became available that allow the automated synthesis of covalent trisoligo junctions bearing either identical or nonidentical sequences [7-10]. Using these technologies it is now possible to work on the reverse strategy, namely, to self-assemble nanostructures from sets of covalent trisoligo junctions. Complementary trisoligo junctions with three identical sequences were shown to self-assemble into nanoobjects having the topology of acetylene and cyclobutadiene if one understands a DNA-double-strand as the noncovalent topological analogon of a C-C-bond (Fig. 2) [9]. It was also shown that kinetic control—applied by means of rapid cooling during hybridization—favors the formation of small and defined nanoobjects instead of large "polymeric" networks of noncovalent superstructures [9]. Very recently, a feasibility study on the issue (2) of this proposal proved successful. It was demonstrated that a tetrahedral nanoobject self-assembles from four trisoligo junctions each having three individual sequences to give a total of $4 \times 3=12$ noncovalent interaction elements (Fig. 3) [10]. Pairing of these sequences yielded six double-stranded "bonds" encoding the edges of the tetrahedron. Gel electrophoresis studies on the assembly and enzymatic digestion of the tetrahedron and its noncovalent intermediates confirmed that all six planned bonds were formed. Circular dichroism studies indicated a B-DNA conformation of the duplex bonds while UV-melting studies revealed the expected melting cooperativity. Distance probing between the nodes of the tetrahedron using fluorescence resonance energy transfer (FRET) gave evidence for equal bond length as to be expected for a tetrahedral geometry. The latter studies also showed that the tetrahedron can act as a noncovalent nanoscaffold for an arraying of functional modules such as dyes in a defined 3D arrangement [10].

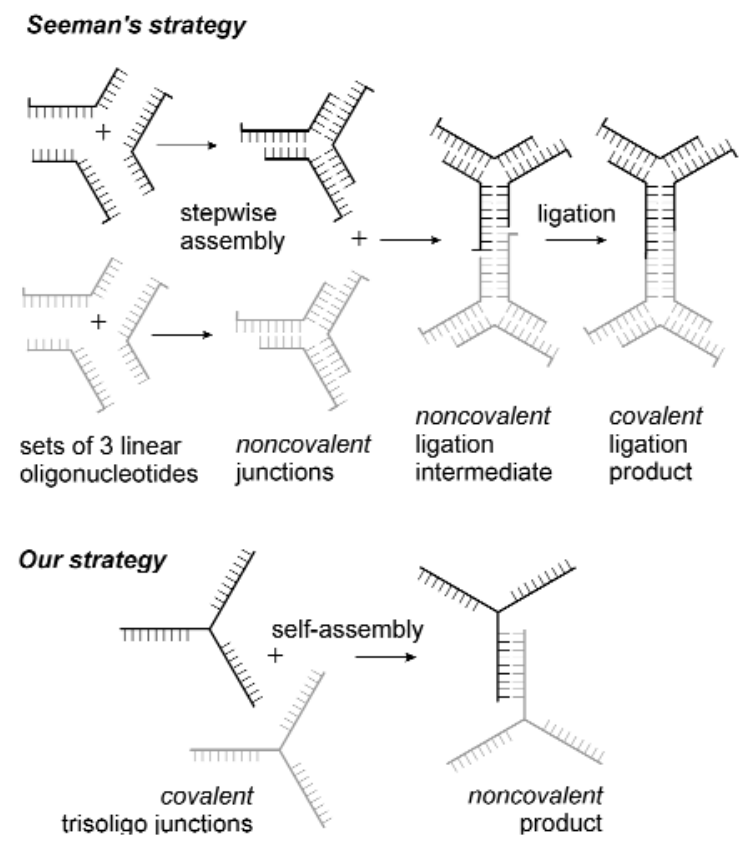

Fig. 1 Two strategies for the construction of DNA-nanoobjects. Seeman's strategy is based on the synthesis of covalent objects from noncovalent junctions. Our strategy involves covalent junctions whose self-assembly leads to noncovalent objects. 

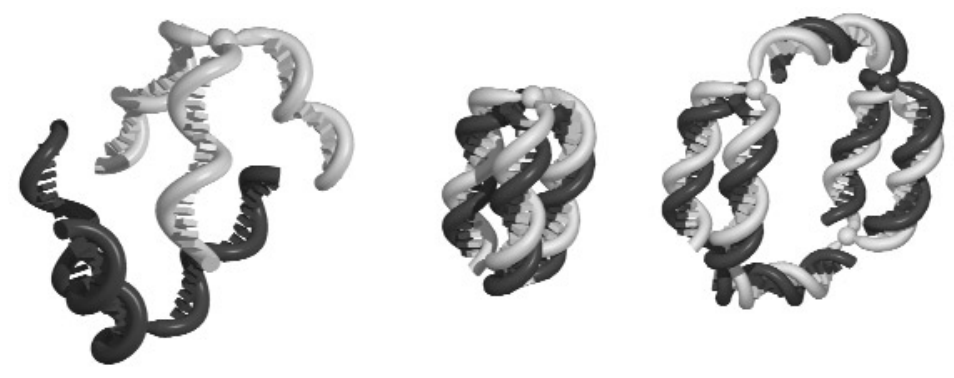

Fig. 2 Self-assembly of two complementary trisoligo junctions (left) yields objects with the topology of acetylene (middle) and cyclobutadiene (right). Kinetic control during self-assembly was shown to favor the acetylene topology.
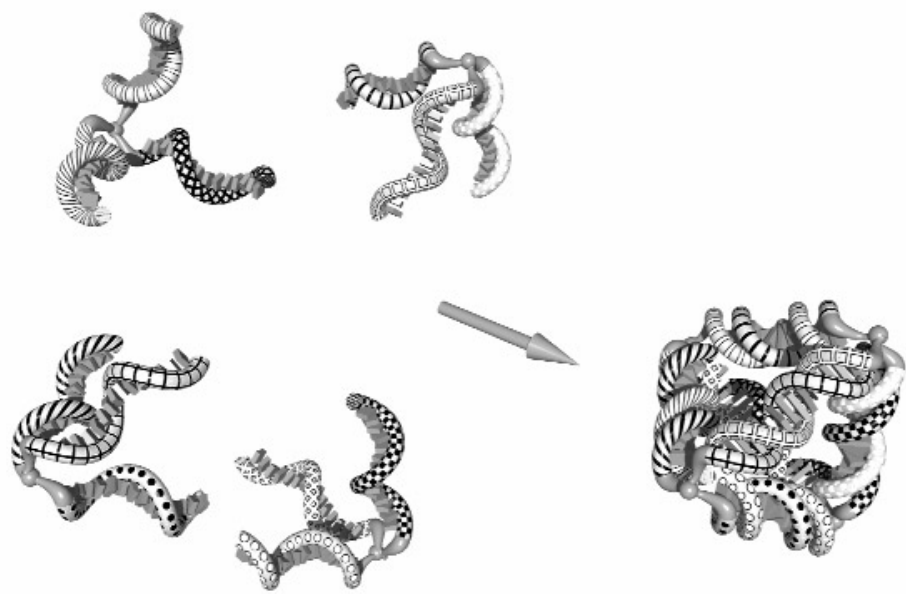

Fig. 3 Self-assembly of a tetrahedral nanoobject from four trisoligo junctions.

\section{Tensegrity and maximal instruction as the keys for nanoarchitecture control}

A tetrahedal scaffold is the smallest object where the topology enforces a "three-dimensional" geometry on the nanometer scale. Generally, we understand trisoligo nanoscaffolds as constructs having rigid DNA-double-stranded "sticks" that are connected by flexible linker "threats". So long as the flexible units are small as compared to the rigid ones, such nanoscaffolds are either stiff in the sense that topology/connectivity determines geometry or not stiff. A cube-shaped scaffold, for example, is not stiff because it can fold in many different ways. In the macroscopic world, a cube-shaped scaffold may even collapse under the influence of gravity, whereas a tetrahedral scaffold does not. Stiff scaffolds maintain their geometric shape and will even stiffen if a force is applied. R. Buckminster Fuller introduced the term "tensegrity" to describe the phenomenon of (geometrical) integrity upon tension and it was recently outlined that this concept has implications for the molecular world [11]. Another issue of nanoscaffold engineering is based on Lehn's "Instructed mixture paradigm" [12]. Lehn coined the latter term for a mixture of interacting molecules where the overall supramolecular structure(s) is(are) instructed by a number of selective interactions in the set of building blocks. There are, however, different ways to instruct the mixture. One should propose to refer to "maximal instruction" if one deals with a set of building blocks, whose self-assembly leads to the target superstructure as the smallest member in a family of conceivable alternatives. It was already shown that noncovalent synthesis can be steered to favor the smallest superstructure by rapid cooling as a means to apply kinetic control [9]. For any given trisoligo nanoscaffold, the maximally instructed set is the one where each corner belongs to an 


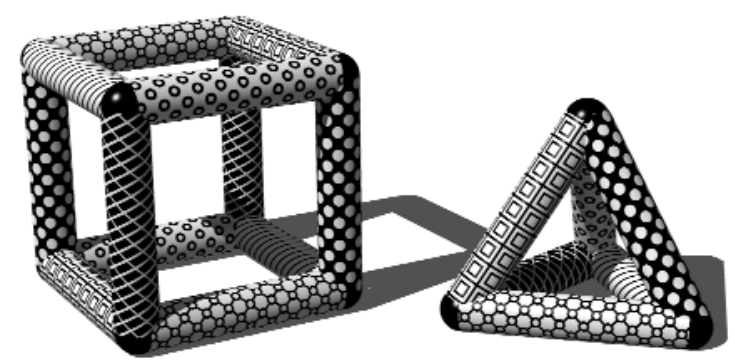

Fig. 4 The tetrahedron, unlike the cube is an example for a nanoobject with (a) stiff tensegrity and (b) maximal instruction. Each texture in the objects indicate an individual double-stranded sequence.

individual trisoligo and each edge to an individual double-stranded sequence (Fig. 4). The maximally instructed set for a tetrahedral nanoscaffold consists of 4 trisoligos, each with 3 different sequences pairing to encode 6 doublestranded bonds. If pairing of all sequences in the set takes place, the noncovalent products may be composed of 4 molecules (tetrahedron), 8 (cube), 12 (truncated tetrahedron), and so on.

\section{Chemical copying of connectivity information (CCC) as the key for nanomachine replication}

We consider connectivity information as a central concept in the design of replicatable nanomachines. For systems with stiff tensegrity, the whole scaffold nanoarchitecture is encoded in the connectivity information, by which each junction "knows" where to integrate during the self-assembly process. Very recently it was shown that the connectivity information of one kind of such junctions can be copied by chemical means (Fig. 5) [13]. Copying of connectivity information is based on a template-directed trislinking reaction, in which the trisoligo junction binds three linear complements, whose $5^{\prime}$-ends come into close spatial proximity and thus can be connected by a suitable trislinking reagent. One should mention at this point that there are two kinds of trisoligo junctions, so-called 3 '-trisoligos where the $3^{\prime}$-ends are connected and so-called $5^{\prime}$-trisoligos with a connection between the $5^{\prime}$-ends. Currently, we know that $3^{\prime}$-trisoligo junctions can be used as templates for the fabrication of $5^{\prime}$-trisoligo junctions.

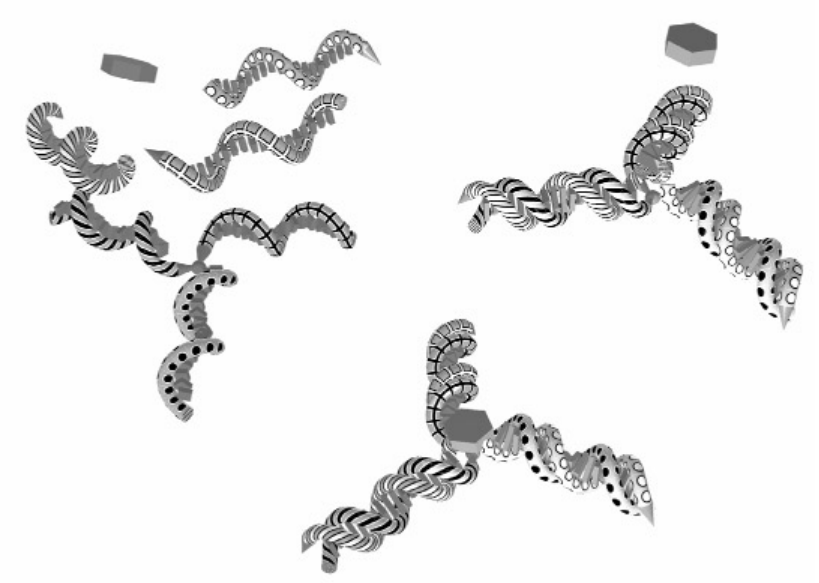

Fig. 5 Illustration of chemical connectivity copying (CCC) by template-directed trislinking. Left: The copying process involves a 3 '-trisoligonucleotidyl with three individually defined sequences, three linear complements whose $5^{\prime}$-ends are drawn as blunt ends, and a trislinker shown as a honeycomb cap. Right: Hybridization leads to a quarter-molecular complex in which the blunt ends come into close spatial proximity. Bottom: The trislinker has reacted to connect the $5^{\prime}$-ends of the copy. 
The proof of replicability of a nanoscaffolded machinery requires also the demonstration of the reverse process which has not been implemented so far. Presently it seems, however, that it is just a matter of a short period of time until a demonstration of a full copying cycle has been achieved.

\section{Cloning and copying on surfaces using eSPREAD}

SPREAD was introduced as a general procedure for the replication of DNA-like materials [14]. The procedure, originally invented to overcome the issue of product inhibition in artificial self-replicating systems, has a special potential for nonnatural modes of copying such as CCC, for which no biotechnological tools such as polymerases or ligases are available. As such and because of its generality, we see this procedure as a key method for nanomachine replication. An electronic chip variant of the procedure (Fig. 6) became the issue of a patent [15]. We propose to develop the eSPREAD procedure (also called cloning and amplification technology, CAT) for the cloning and copying of nanomachines on the surface of such electrophoresis chips.

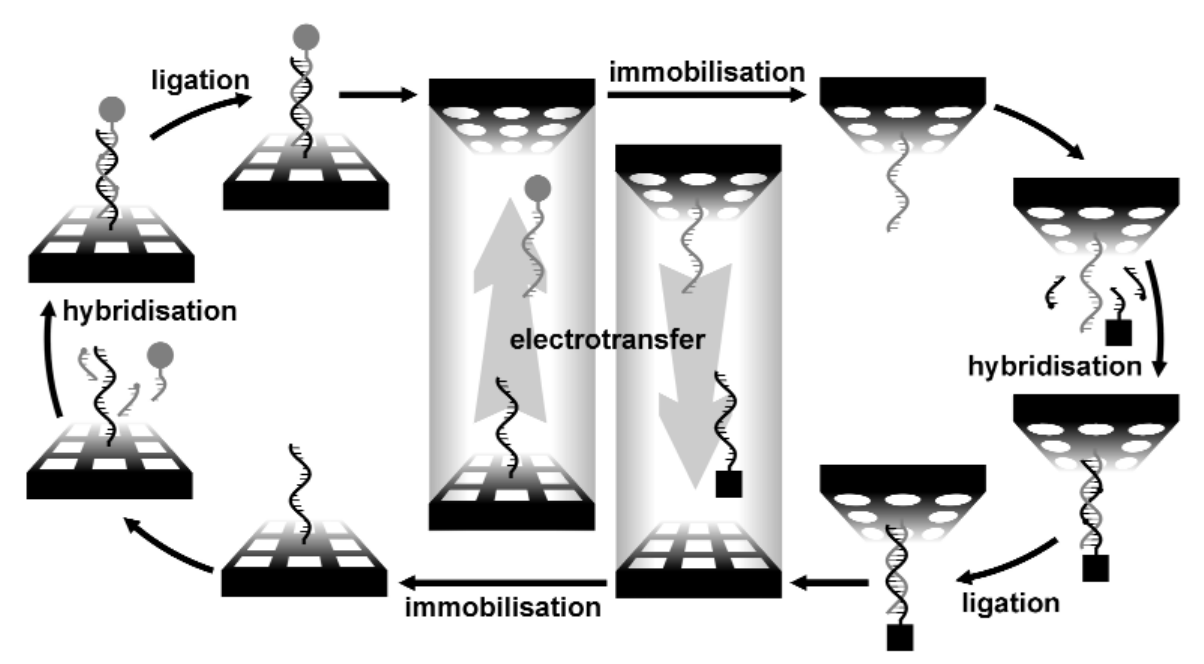

Fig. 6 The eSPREAD-procedure makes use of two planparallel electrodes to which permeation layers allowing for two orthogonal immobilization chemistries (circles/squares) are attached. A template is bound to the lower electrode. Hybridization and ligation of complementary fragments yields a copy, which is transferred to an adjacent site of the counterelectrode by the help of an electric field. The copy is immobilized to become a template for the production of its complement which is transferred to the lower electrode by reversing the electric field. eSPREAD thus proceeds with the conservation of sequential and spatial information enabling it to copy and to clone template molecules by a flip-flop between the two electrodes.

\section{Linear conjugates as building blocks for junction eSPREADing and nanoscaffolded multifunctionality}

We propose modular monoconjugates as construction elements for junction replication and amplification using eSPREAD (introduced in step 4 of Fig. 7). The scheme proceeds from triple-function $3^{\prime}$-trisoligo templates where one of the three functions is needed for trisoligo template immobilization. The copy product of steps $1-3$ is a $5^{\prime}$-trisoligo that is used as a template in the second half of the cycle. Any modular function that is (a) conjugable to a linear oligonucleotide, and (b) compatible with the implementation protocol of eSPREAD may be utilized here. As such, peptides, cyclopeptides, proteins, sacharides and oligosacharides, dyes, organic conductors and semiconductors, lipids, steroids, dendrimers, fullarenes, nanotubes, as well as inorganic nanocrystals, clusters, and nanoparticles may become 
the issue of nanoscaffolded multifunctionality. Moreover, because CCC is a purely chemical process, the informational material from which the scaffold is self-assembled does not necessarily need to be based on oligonucleotides. Any functional mimic, such as "spiegelmers" [16], PNA [17], pRNA [18], and many more may replace the oligonucleotide part of the scaffold. Figure 8 illustrates the self-assembly process using a single triple-function trisoligo. Not only 3 but all of the 12 sequences in the 4 trisoligos of a tetrahedral nanoscaffold may bear an individual function module.

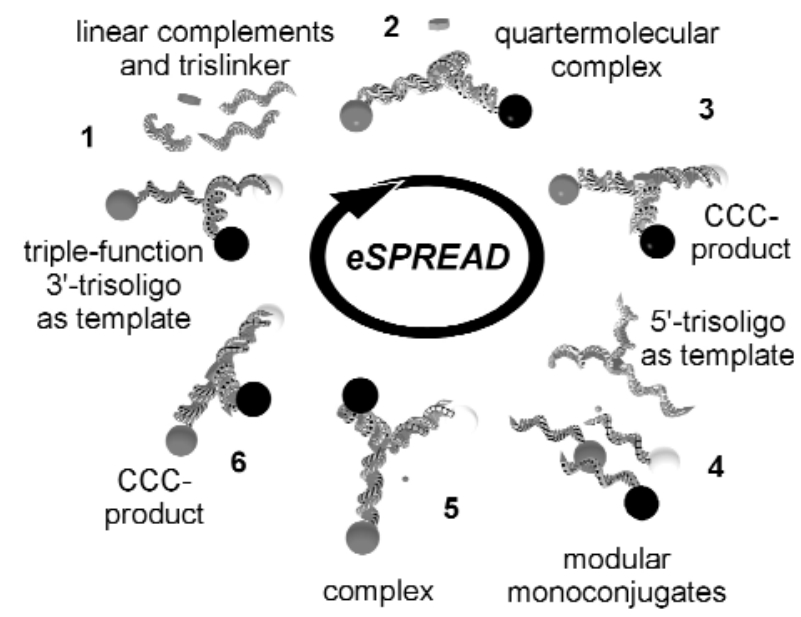

Fig. 7 eSPREADing the connectivity information of a triple-function 3 '-trisoligo template (1). The copying and amplification process involves modular monoconjugates (4) as basic construction elements. The three functions per junction are indicated by balls with different grey shades.

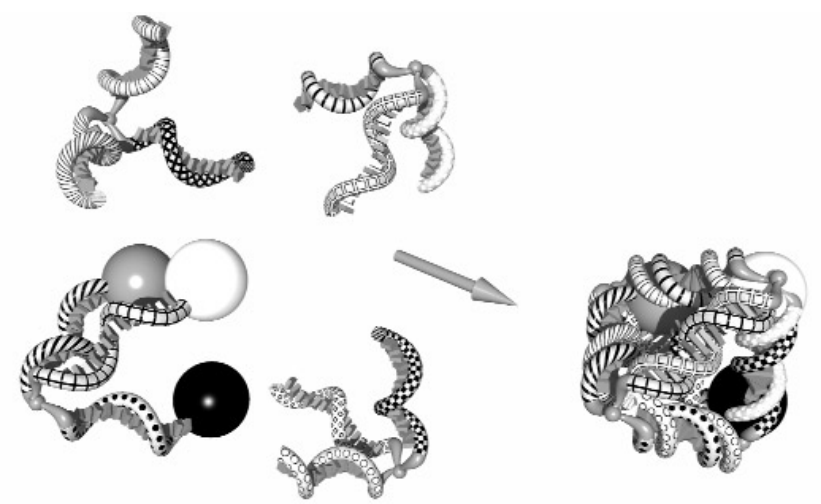

Fig. 8 Incorporation of a triple-function $3^{\prime}$-trisoligo during the self-assembly process.

\section{Directed evolution of replicatable nanomachines}

Once eSPREADing of connectivity information has led to successful protocols for junction amplification, there is only a small step toward the directed in vitro evolution of multifunctional nanoscaffolded machinery. Starting from modular monoconjugates as basic construction elements, random connectivity information could be seeded by statistical, viz., nontemplated synthesis of triple-functionalized junctions. Statistically, when starting from 12 monoconjugates, there are 12 possible homotrimers, $12 \times 11=132$ possible heterotrimers with two different arms, and $12 \times 11 \times 10=1320$ trisoligos with three different arms. As such, statistics clearly favors multifunctionality. If one asks for the total num- 
ber of possible arrangements on a tetrahedral scaffold, one may conceive of connecting the four corners of the tetrahedron with the six duplex bonds, each carrying two modules. After fixing the first bond, there are five distinguishable locations to place the second bond, however, each in two different orientations. For the third bond there are four possibilities again in two orientations. The next bond comes up with three possibilities in two orientations and so on. So the total number of arrangements is $5 ! \times 2^{5}=3840$. Generally, a spherically closed object with $\mathrm{n}$ bonds has $2^{n-1} \times(n-1)$ ! possible arrangements. A nanoscaffold with dodecahedrane topology $(n=30)$, for example, could already exist in $4.7 \times 10^{39}$ configurations. One may argue that the functional diversity of such constructs is lower due to symmetry reasons. This argument however may hold only for ideal geometries with equal bond lengths. Once a set of trisoligo building blocks with different arm lengths is statistically assembled, geometrically distorted objects will result that may have the full repertoire of multifunctionality. Multifunction probing may proceed similarily as the SELEX procedure [19], where a random population of linear RNA and DNA molecules undergoes a series of selection and amplification steps finally leading to a rather uniform population of molecules that fulfills the requested function in an optimal way. An illustration of the procedure is shown in Fig. 9.
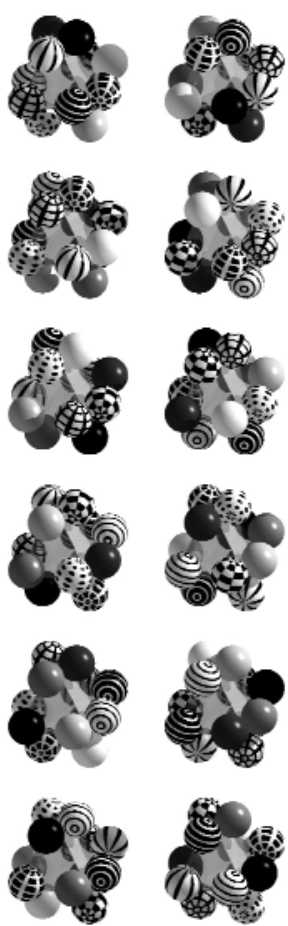
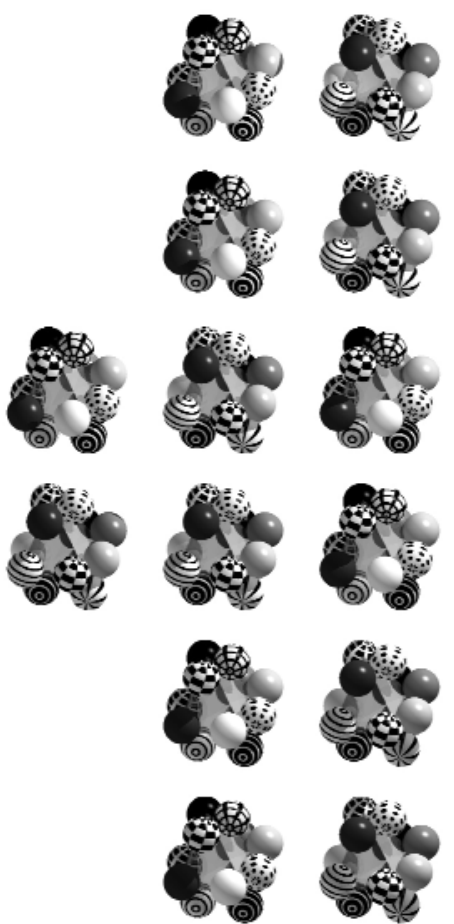
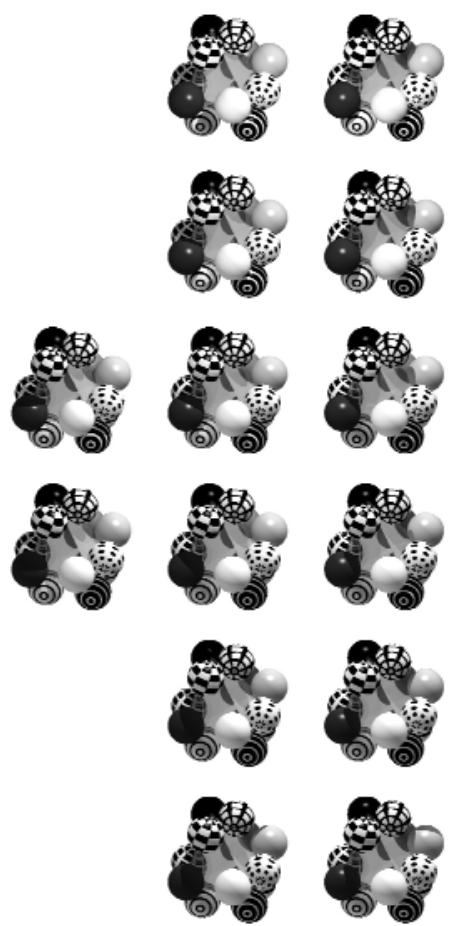

Fig. 9 Illustration of a directed evolution process of replicatable nanomachines based on a tetrahedral scaffold. A population of 12 arrangements (left) is probed to suit a given complex function, e.g., to bind to a cluster of receptors on the surface of a cell. Those constructs ( 2 here) that fulfill the task are selected from the initial population by chemical means, e.g., by affinity chromatography, and then amplified to arrive at a new population, in which the functional constructs are enriched (middle). Another round of selection and amplification using more stringent conditions finally leads to a (more or less) uniform population of nanomachines with an optimal array of function modules. 


\section{Probing the existence of nanoepitopes on the surface of biological cells}

Among the possible tasks for such constructs are catalysis, binding, and sensing tasks. While the gross geometry of a nanoscaffold with stiff tensegrity seems to be predictable, the detailed orientation and positioning of the modules may be more difficult to control. Depending on the nature of the module itself, the linker between the module and the scaffold, as well as the lengths of the junction arms, modules may prefer to orient themselves into the interior or the exterior of the scaffold. While catalysis and small molecule binding tasks may benefit from an endopresentation of modules, binding to objects larger than the scaffold will benefit from their exopresentation. A rather appealing target for the application of such constructs is the surface of biological cells. Little is known to what extent the arrangement of individual cell-surface molecules and receptors such as integrins, G-protein coupled receptors, lectins, ion channels, and others are spatially organized by the cytoskeleton and to what extent these molecules are presented in a statistical arrangement. If the cytoskeleton "imprints" 2D spatial information to the outside of the cell, this arrangement may vary with the state of cell cycle as well as a function of cell differentiation. Nanoepitope screening through the arraying of the ligands of the cell-surface molecules via an artificial nanoscaffold may shed new light on the classification of cell types and tissues. If cell types including cancer cells can be indeed recognized by cooperative binding to a number of different modules arrayed on the scaffolds, it seems conceivable to load the scaffolds with additional "therapy" modules. Especially, modules with the function to alert the immune system, or, metallic nanoparticles used to dissipate heat upon receival of external radiation seem to be promising candidates for the stepwise implementation of medical nanomachines.

\section{External control of the operation of such nanomachines by $\mathrm{GHz}$ radio frequency magnetic field inductive heating of metal clusters attached to such constructs}

The remote control of the operation of nanomachines may be based on an induced conformational change. The latter may affect, e.g., the activation of modules for a remotely controlled action, or the arrangement of modules (exo vs. endopresentation). External control may be applied by a change of temperature, light, $\mathrm{pH}$, ionic strength, as well as the concentration of specific factors (e.g., heavy metal ions) for which selective recognition modules may be loaded onto the scaffolds. If one, however, considers a potential use of such nanomachines inside the human body all of the above control parameters are only applicable in limited cases. Most elegant in this context is, however, the possibility to switch conformations by the dissipation of heat from an inductive heating of metallic or magnetic nanoparticles attached to such constructs. Very recently it was shown that a gold nanocrystal $(1.4 \mathrm{~nm})$ can act as a nanoscale antenna for the receival of the magnetic component of $\mathrm{GHz}$ radio frequency radiation [20]. It was demonstrated that irradiation of a gold-labeled molecular beacon leads to its opening, while no opening happened in the absence of the gold-cluster label attached. The results indicated that this technique can be used to heat up single molecules [20]. The technique of single-molecule heating may soon go beyond its proof-of-concept stage, once thermostable gold-cluster labels become available. A new generation of biocompatible gold clusters, tailored for nanotechnological, biomolecular, and nanomedical applications, will be introduced soon. The material (called RUBiGold) is based on a robotic grip design for the packaging of the gold-cluster core (Fig. 10) [21]; its thermostability is orders of magnitude higher than that of the currently available material. Current research in this field is devoted to the development of bioconjugable nanowires, nanocircles, and nanocoils having a precisely defined size. The length of such constructs is expected to control the degree of single-molecule heating. 


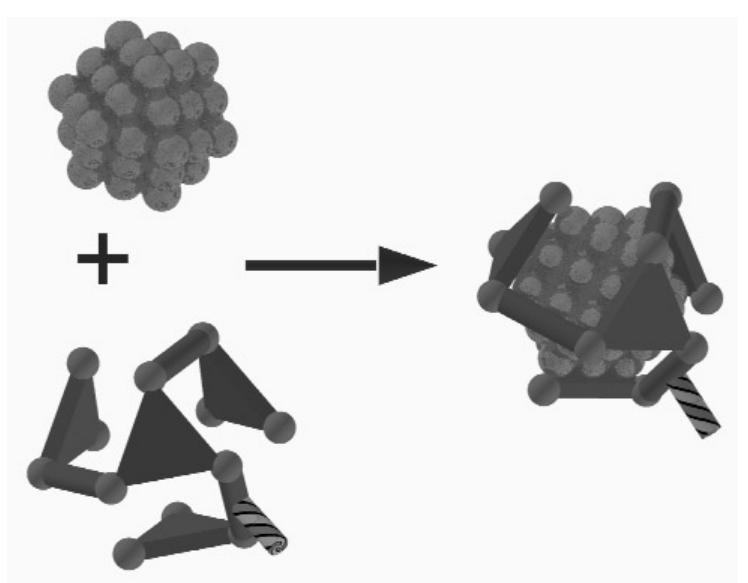

Fig. 10 RUBiGold is based on a single dodecadentate thioether grip carrying a functional moiety (candystick texture) that allows the monoconjugation of gripped gold cluster $(1.4 \mathrm{~nm})$ to biomolecules and other materials.

\section{CONCLUSION}

The vision of self-replicating nanorobots as introduced by Drexler is based on the concept of a generalpurpose nanomechanical assembler that builds any molecule and material including itself in an atomby-atom fashion. It underestimates the individuality and diversity of chemical behavior and implies an unfruitful disappreciation of chemistry as a science and of chemical synthesis as an art. Being able to use an AFM tip to move around xenon atoms on the surface of a single crystal to write a company's logo is indeed a remarkable achievement, but from here to the nanomechanical synthesis of objects shaped into the diamond lattice with atomic precision is a very long way. Nevertheless, Drexler's vision should not be abandoned because it holds a number of promising ideas of where to go and what to do in the future. Drexler's core idea of self-replicating nanorobots, however, may be challenged by alternative and more natural concepts of implementation such as outlined in this manifesto. Chemistry has been looking into this direction for a number of years-the first demonstration of a chemical self-replicating system [22] and even a cautiously hidden claim to go for self-reproducing molecular robots [23] were reported in the same year when Drexler's book came out. In a technical report published by Iben Browning as early as 1956 and reprinted in 1978, future chemistry was seen as a central science facing two challenges, namely, the deciphering of the origin-of-life problem and the creation of artificial selfreproducing molecular machinery. It seems that this future has arrived today.

\section{ACKNOWLEDGMENTS}

This work was supported by Deutsche Forschungsgemeinschaft (SFB-452), Bundesministerium für Bildung und Forschung (Bionanotechnologie), and Fonds der Chemischen Industrie. We thank Michael Wüstefeld for DNA synthesis, Rolf Breuckmann for MALDI and ESI analysis, and Beate Materne and Carsten Lodwig for technical assistence.

\section{REFERENCES}

1. N. Winkless and E. Browning. Robots on Your Doorstep. Robotics Press, Portland (1978).

2. K. E. Drexler. Engines of Creation: The Coming Era of Nanotechnology. Anchor Press, Doubleday, New York (1986).

3. R. E. Smalley. Sci. Am. 285 (3), 76-77 (2001).

4. G. M. Whitesides. Sci. Am. 285 (3), 78-83 (2001). 
5. Overview: C. M. Niemeyer. Angew. Chem., Int. Ed. 40, 4128-4158 (2001).

6. J. Chen and N. C. Seeman. Nature 350, 631-633 (1991).

7. S. Jordan. Synthesis of oligonucleotide building blocks for self-replication and self-assembly experiments (in German), thesis, Göttingen (1993).

8. M. S. Shchepinov, K. U. Mir, J. K. Elder, M. D. Frank-Kamenetskii, E. M. Southern. Nucleic Acids Res. 27, 3035-41 (1999).

9. M.Scheffler, A. Dorenbeck, S. Jordan, M. Wüstefeld, G. von Kiedrowski. Angew. Chem., Int. Ed. 38, 3311-3315 (1999).

10. A. Dorenbeck, M. Scheffler, M. Wüstefeld, G. von Kiedrowski. Submitted for publication. See also A. Dorenbeck. DNA nanostructures by self-assembly of trisoligonucleotidyls (in German), thesis, Bochum (2000); <http://www-brs.ub.ruhr-uni-bochum.de/netahtml/HSS/Diss/DorenbeckAxel/>.

11. D. E. Ingber. Ann. Rev. Physiol. 59, 575-599 (1997).

12. J. M. Lehn. Supramolecular Chemistry-Concepts and Perspectives, VCH, Weinheim (1994).

13. L. Eckardt, K. Naumann, W. M. Pankau, M. Rein, M. Schweitzer, N. Windhab, G. von Kiedrowski. Nature 420, 286 (2002).

14. A. Luther, R. Brandsch, G. von Kiedrowski. Nature 396, 245-248 (1998).

15. J. P. Fürste, S. Klussman, T. Klein, G. von Kiedrowski. Cloning and copying on surfaces, EP1135527, WO0032809, US6534271, DE19854946.

16. A. Nolte, S. Klussmann, R. Bald, V. A. Erdmann, J. P. Fürste. Nature Biotech. 14, 1116-1119 (1996).

17. P. E. Nielsen, M. Egholm, R. H. Berg, O. Buchard. Science 254, 1497-1500 (1991).

18. S. Pitsch, S. Wendeborn, B. Jaun, A. Eschenmoser. Helv. Chim. Acta 76, 2161-2183 (1993).

19. C. Tuerk and L. Gold. Science 249, 505-510 (1990).

20. K. Hamad-Schifferli, J. J. Schwartz, A. T. Santos, S. Zhang, J. M. Jacobson. Nature 415, 152-155 (2002).

21. W. M. Pankau, S. Mönninghoff, G. von Kiedrowski. Thermostable and monoconjugable gold cluster by gripping with a dodekadentate thioether ligand. Submitted, patent pending; see also W. M. Pankau, K. Verloist, G. von Kiedrowski. Chem. Commun. 519-520 (2001).

22. G. von Kiedrowski. Angew. Chem., Intl. Ed. Engl. 25, 932-935 (1986).

23. G. von Kiedrowski. Orig. Life Evol. Biosph. 16, 468 (1986). 